\title{
Prognostic Role of Serum Alpha-Fetoprotein in Hepatocellular Carcinoma Patients with Radiofrequency Ablation
}

\author{
Mohamed O Khalifa ${ }^{1}$, Ossama A Ahmed ${ }^{2}$, Eslam Safwat ${ }^{2}$ \\ ${ }^{I}$ Tropical Medicine Department, Ain Shams University, Cairo, Egypt \\ ${ }^{2}$ Internal Medicine Department, Ain Shams University, Cairo, Egypt
}

Corresponding Author Mohamed Omar

Khalifa

Mobile:

+20222871187

E mail:

dmohammedomar@

hotmail.com

Key words: Alpha-fetoprotein, Hepatocellular

carcinoma, Prognosis, Overall survival, Recurrence, Diseasefree
Background and study aim: Prognostic value of serum alpha-fetoprotein (AFP) in hepatocellular carcinoma (HCC) is still debatable. We aimed to study this role in HCC patients who underwent radiofrequency ablation (RFA).

Materials and Methods: Records from HCC patients were retrospectively analyzed between January 2012 and December 2016. A minimum data set for each patient record of a follow-up period of at least 1 year was pre-defined before enrollment. In all, 153 patients were enrolled. AFP levels were recorded for all patients at the time of diagnosis, 1 month after RFA and at 3-month intervals afterward. Patients were divided according to pretreatment AFP level into 3 groups: group 1: AFP $<20 \mathrm{ng} / \mathrm{mL}$, group 2: AFP 20-200 ng/mL and group 3: AFP > $200 \mathrm{ng} / \mathrm{mL}$.
Results: Pretreatment AFP is not significantly correlated with age, baseline lesion number or size, baseline Child score or class, post RFA recurrence or death. The overall survival rates were $95 \%$, $75.6 \%, 55.6 \%, 48.8 \%$, and $48.8 \%$ at $1,2,3,4$, and 5 years respectively. On comparing the 3 groups on disease-free survival, there was no statistically significant difference among the three classes. Child class A patients showed statistically significant better survival after RFA than those with Child class B. The ROC curve showed that AFP had inadequate accuracy to discriminate survivors and deceased patients and to discriminate patients with recurrence from those without recurrence.

Conclusion: AFP level could not be used as a good predictor of either death or recurrence of HCC after RFA

\section{INTRODUCTION}

Alpha-fetoprotein (AFP) is considered the most thoroughly investigated marker for diagnosing hepatocellular carcinoma (HCC). However, it has a limited diagnostic performance for the surveillance of HCC. Two reasons may explain this; first, high AFP levels could be seen in patients with chronic hepatitis and liver cirrhosis [1], second, only a small proportion of early-stage HCCs (10-20\%) present with increased AFP levels [2].

The American Association for the Study of Liver Diseases (AASLD) guidelines for HCC diagnosis and treatment, however, has recently eliminated AFP measurement from the surveillance armamentarium because of its poor sensitivity and specificity for the diagnosis of HCC [3].

Also, AFP assessment is not included in The Barcelona Clinic Liver Cancer (BCLC) classification system, although it has been identified by several studies as an overall independent predictor of survival [4].

However, most of studies about the prognostic value of AFP have included heterogeneous cohorts of patients, thus preventing a proper evaluation of its performance as a prognostic marker in a selected subset of patients [5].

In this study, we aimed at evaluating the prognostic role of AFP in patients with HCC treated with radiofrequency ablation (RFA). 


\section{MATERIALS AND METHODS}

This retrospective study was conducted at the HCC and Hepatology clinics of the Tropical and Internal Medicine Departments, Ain Shams University Hospitals, Cairo, Egypt.

All patients with HCC who were diagnosed and underwent radiofrequency ablation in the period between January 2012 and December 2016 were reviewed and the data from the patients who fulfilled the inclusion criteria were retrospectively retrieved from their files.

The minimum data set within the patient record with a 1-year follow-up period was predefined before collection of data to be included as a record in this retrospective study. This study was confirmed to meet the standards of the Declaration of Helsinki and current ethical guidelines and was approved by the Research and Ethics Committee of Ain Shams University, Cairo, Egypt, in accordance with local research governance requirements.

Incomplete files or patients who did not complete a follow-up period of 1 year were excluded from the study.

The main characteristics of the database have been previously reported. Our database includes patient demographics, main biochemical and hematological parameters, etiology and stage of liver disease, the presence of comorbidities, baseline and serial measurements of AFP, HCC stage and treatment, patient survival, and mortality.

Among all treated HCC patients from January 2012 to December 2016, patients who fulfill the following criteria were only included:

1. Patient's age $>18$ years,

2. Child-Pugh class A and B cirrhosis,

3. Confirmed diagnosis of $\mathrm{HCC}$ according to AASLD guidelines [3],

4. Who underwent RFA for HCC depending on the BCLC staging system with no eligibility to undergo liver transplantation or resection, and

5. Who achieved complete tumor response 1 month after RFA according to modified RESICT criteria [6], i.e. complete disappearance of intra-tumoral enhancement in all target lesions using dynamic imaging; either CT or MRI.

Patients with advanced liver disease (Child-Pugh class C) or those with extra-hepatic metastasis or gross vascular invasion and those patients with any previous HCC treatment were excluded from this study.
We calculated Disease-free survival (DFS) from the time of complete response to a curative procedure to the time of disease recurrence. Overall survival (OS) was calculated from the time of intervention to the date of death or that of the last follow-up visit (December 2017).

Analysis of survival was done yearly after treatment. The maximum tumor diameter was the proposed tumor size. In case of multiple tumors, the size was measured as the sum of the maximum diameters of all tumors.

Follow up of all patients, with the measurement of serum AFP and CTs, was done every 3 months in the first year after treatment, and then every 6 months for the next 4 years.

Patients' informed consent to the study was not a requirement because their records were reviewed retrospectively and the clinical data that were obtained after each patient agreed to RFA by informed written consent before intervention.

\section{Statistical analysis:}

Statistical analysis was performed with SPSS software (SPSS Inc., Chicago, IL, USA). Data were expressed as the mean $\pm \mathrm{SD}$, median or count and percentage. Differences in continuous variables between the different groups' data were assessed by independent t-test. Mann-Whitney $U$ tests, Kruskal-Wallis tests or $\chi 2$ tests were used to compare non-parametric variables. A level of significance (p) less than 0.05 was significant. One-way analysis of variance (ANOVA) was applied to compare all groups on quantitative variables to determine significant differences. Pearson correlations were used to assess the correlation between parameters of interest. Pearson correlation coefficients point to a direct correlation, while negative values point to an inverse correlation and were considered significant at the 0.05 level. Univariate regression analysis was used to assess the correlations of the predictors of death or recurrence. Life tables and KaplanMeier curves were used to present survival. The log-rank test was used to compare survival times between the different groups.

The probability of the AFP level predicting death or recurrence was used to construct receiver operating characteristic (ROC) curve. The efficacy of each panel was assessed by using area under the curve (AUC). As the AUC of AFP predicting death or recurrence did not reach a statistically significant level, no optimal cut-off values were selected. 


\section{RESULTS}

According to pretreatment AFP level, patients were divided into 3 groups; group 1 included 59 patients (49 males and 10 females) with AFP less than $20 \mathrm{ng} / \mathrm{ml}$, group 2 included 54 patients (43 males and 11 females) with AFP levels of 20-200 $\mathrm{ng} / \mathrm{ml}$ and group 3 included 40 patients (30 males and 10 females) with AFP above $200 \mathrm{ng} / \mathrm{ml}$.

Table 1 shows the main demographic, clinical and tumor characteristics of the 153 study patients.

The patients' mean age was $56.43 \pm 7.02$ years, and approximately $80 \%$ of them were males. Hepatitis $\mathrm{C}$ virus was the main underlying etiology of liver cirrhosis $(n=142,92.8 \%)$. Around twothirds of the patients were of Child-Pugh class A, and three-quarters of the patients had a single lesion.

The maximum diameter of the HCC lesion was $\geq$ $3 \mathrm{~cm}$ in 124 patients $(81 \%)$. Serum AFP levels were within the normal range $(<20 \mathrm{ng} / \mathrm{ml})$ in 59 patients $(38.6 \%)$, mildly elevated $(20-200 \mathrm{ng} / \mathrm{ml})$ in 54 patients $(35.3 \%)$, and markedly elevated $(>200 \mathrm{ng} / \mathrm{ml})$ in 40 patients $(26.1 \%)$.

In the present study, tumor recurrence was recorded in 88 cases $(57.5 \%)$, and $53(34.6 \%)$ patients died during follow-up.

Comparison between the 3 groups on gender, age, lesion number, size of the largest lesion, Child class and score, recurrence and death revealed no significant differences between the three groups for any of the parameters as shown in table (2).

The correlation between AFP and other variables (age, size, number of lesions, Child score, and class, recurrence, and death) revealed that pretreatment AFP was not significantly correlated with age, baseline lesion number or size, baseline Child score or class, post RFA recurrence or death.

The overall survival intervals (time to death or end of the study in months), were 95\%, $75.6 \%$,
$55.6 \%, 48.8 \%$, and $48.8 \%$ at $1,2,3,4$ and 5 years, respectively. The mean survival interval was 33.6 months in group 1, 34.3 months in group 2, and 28.6 months in group 3 with no evidence of significant differences between the three groups $(\mathrm{p}=0.207)$.

Figure (1) shows the Kaplan-Meier survival curves of all groups. No significant differences was noticed among the three AFP classes in overall survival $(\chi 2=1.846, \mathrm{P}=0.397)$.

The median recurrence-free interval was 28,28 and 35 months in group 1, 2 and 3; respectively $(\mathrm{p}=0.777)$.

On comparing the 3 groups on disease-free survival, there was insignificant differences among the three AFP classes $(\chi 2=1.859, \mathrm{P}=0.3975)$ as shown in figure (2).

The Kaplan-Meier overall survival curves of the 153 studied patients, subdivided according to their Child class (Child A and Child B) at the time of diagnosis of HCC, revealed that Child class A patients showed a better survival after RFA than those with class $\mathrm{B}(\chi 2=34.613, \mathrm{P}=0.000)$.

The Kaplan-Meier overall survival curves of the studied patients, subdivided according to their lesion size at the time of HCC diagnosis $(<3$ and $>/=3 \mathrm{~cm}$ ), revealed no statistically significant differences $(\chi 2=0.305, P=0.581)$. Similarly, comparison of the patients in their lesion number at the time of diagnosis of HCC (uninodular and multinodular), the Kaplan-Meier survival curves showed insignificant differences $(\chi 2=0.001, \mathrm{P}=$ 0.979).

Alpha-fetoprotein had an inadequate accuracy in discriminating survivors and deceased patients (AUC 0.435, 95\% CI 0.338-0.531) (Figure 3). Also, AFP had an inadequate accuracy to discriminate patients with recurrence from those without recurrence $(\mathrm{AUC}=0.476,95 \%$ CI $0.378-0.573$ ) (Figure 4). 
Table (1): Baseline Characteristics of Patients ( $\mathrm{N}=153)$

\begin{tabular}{|c|c|}
\hline Variable & $\mathbf{N}(\%)$ \\
\hline \multicolumn{2}{|l|}{ Age (years) } \\
\hline Mean \pm SD & $56.43 \pm 7.02$ \\
\hline Range & $42-76$ \\
\hline \multicolumn{2}{|l|}{$\overline{\operatorname{Sex}}$} \\
\hline Male & $122(79.7)$ \\
\hline Female & $31(20.3)$ \\
\hline \multicolumn{2}{|l|}{ Viral infection } \\
\hline HCV positive & $142(92.8)$ \\
\hline HBV positive & $5(3.3)$ \\
\hline HBV/HCV co-infection & $4(2.6)$ \\
\hline Both negative & $2(1.3)$ \\
\hline \multicolumn{2}{|l|}{ Child-Pugh class } \\
\hline Class A & $104(67.9)$ \\
\hline Class B & $49(32.1)$ \\
\hline \multicolumn{2}{|l|}{ Child-Pugh score } \\
\hline 5 & $55(35.9)$ \\
\hline 6 & $49(32.1)$ \\
\hline 7 & $28(18.3)$ \\
\hline 8 & $19(12.4)$ \\
\hline 9 & $2(1.3)$ \\
\hline \multicolumn{2}{|l|}{ Level of AFP (ng/ml) } \\
\hline$<20$ & $59(38.6 \%)$ \\
\hline $20-200$ & $54(35.3 \%)$ \\
\hline$>200$ & $40(26.1 \%)$ \\
\hline \multicolumn{2}{|l|}{ Tumor Characteristics } \\
\hline \multicolumn{2}{|l|}{ Number of lesions } \\
\hline $\begin{array}{c}\text { Single lesion } \\
\end{array}$ & $115(75.2)$ \\
\hline Multiple lesions & $38(24.8)$ \\
\hline \multicolumn{2}{|l|}{ Diameter of the largest lesion $(\mathrm{cm})$} \\
\hline$<3 \mathrm{~cm}$ & $29(19)$ \\
\hline$\geq 3 \mathrm{~cm}$ & $124(81)$ \\
\hline
\end{tabular}

Table (2): Comparison between the 3 groups regarding different parameters

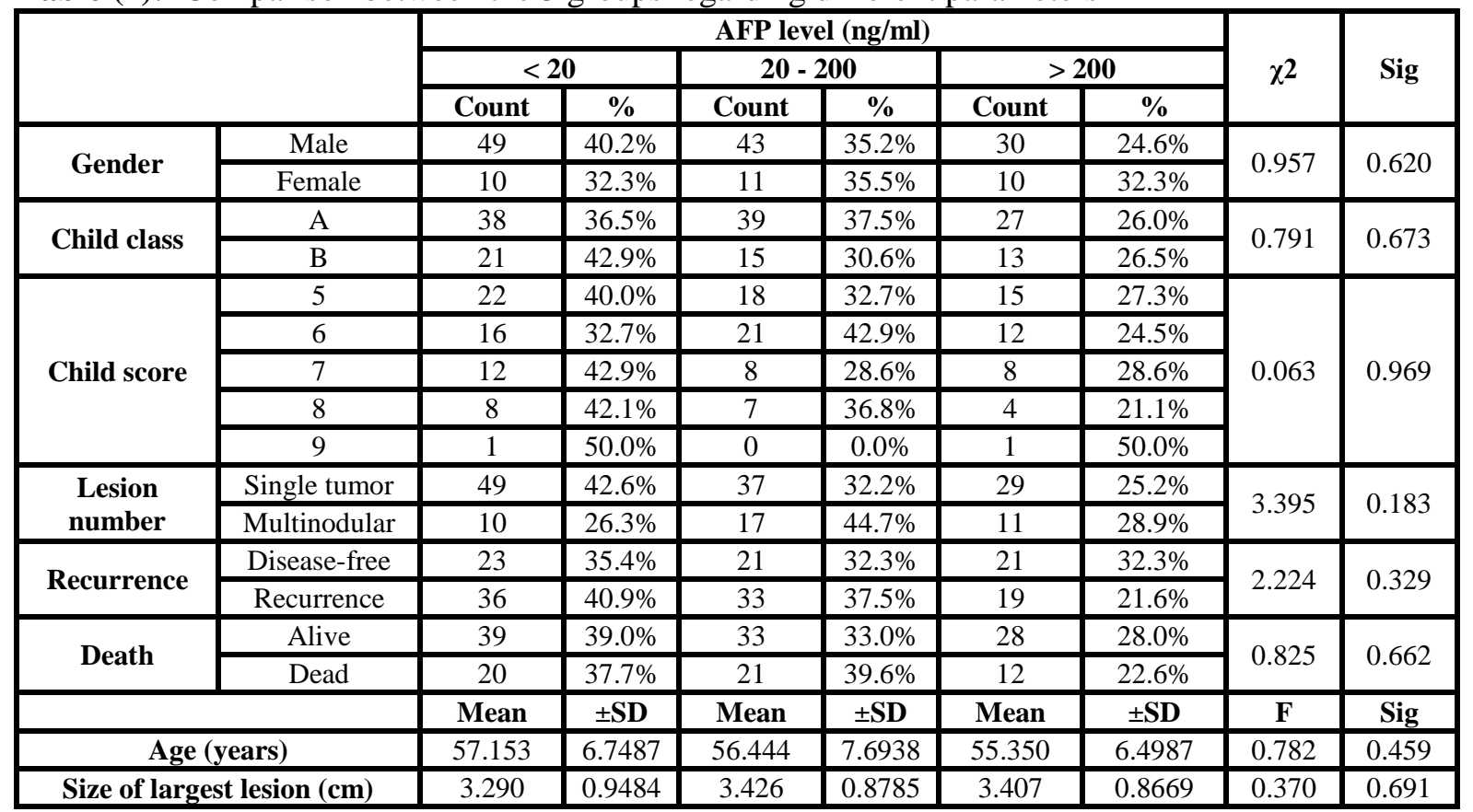

Khalifa et al., Afro-Egypt J Infect Endem Dis 2018; 8(4): 164-173

https://aeji.journals.ekb.eg/

http://mis.zu.edu.eg/ajied/home.aspx 


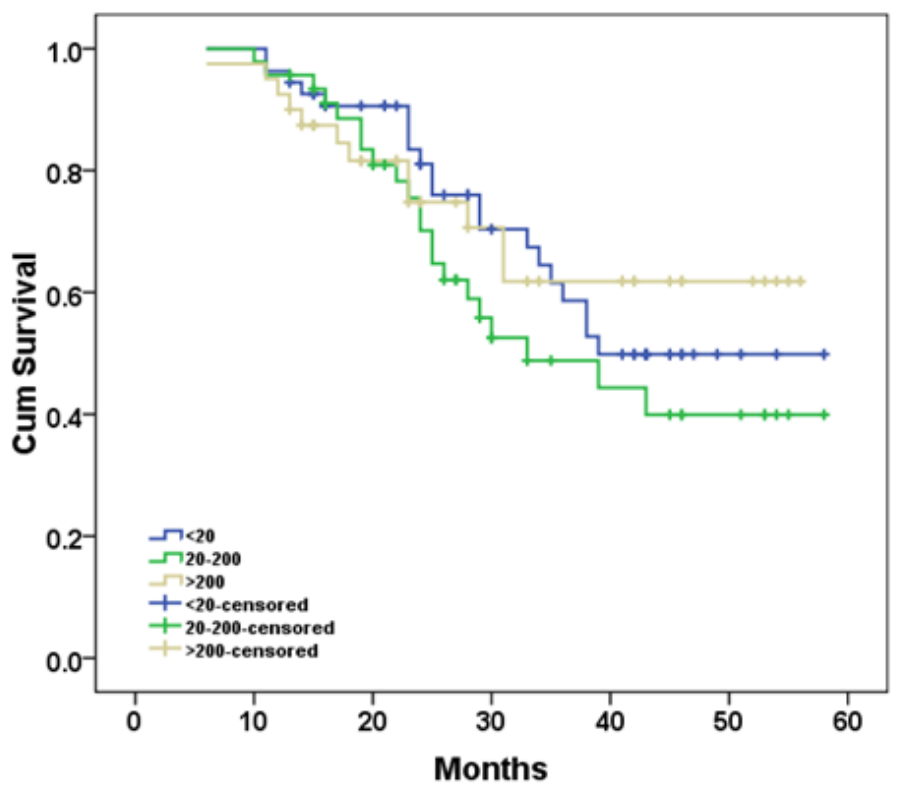

Figure (1): Kaplan-Meier survival curves showing the overall survival of the 153 studied patients subdivided according to their alpha-fetoprotein serum levels at the diagnosis of HCC $(<20 \mathrm{ng} / \mathrm{ml} ; 20$ $200 \mathrm{ng} / \mathrm{ml} ;>200 \mathrm{ng} / \mathrm{ml}$ )

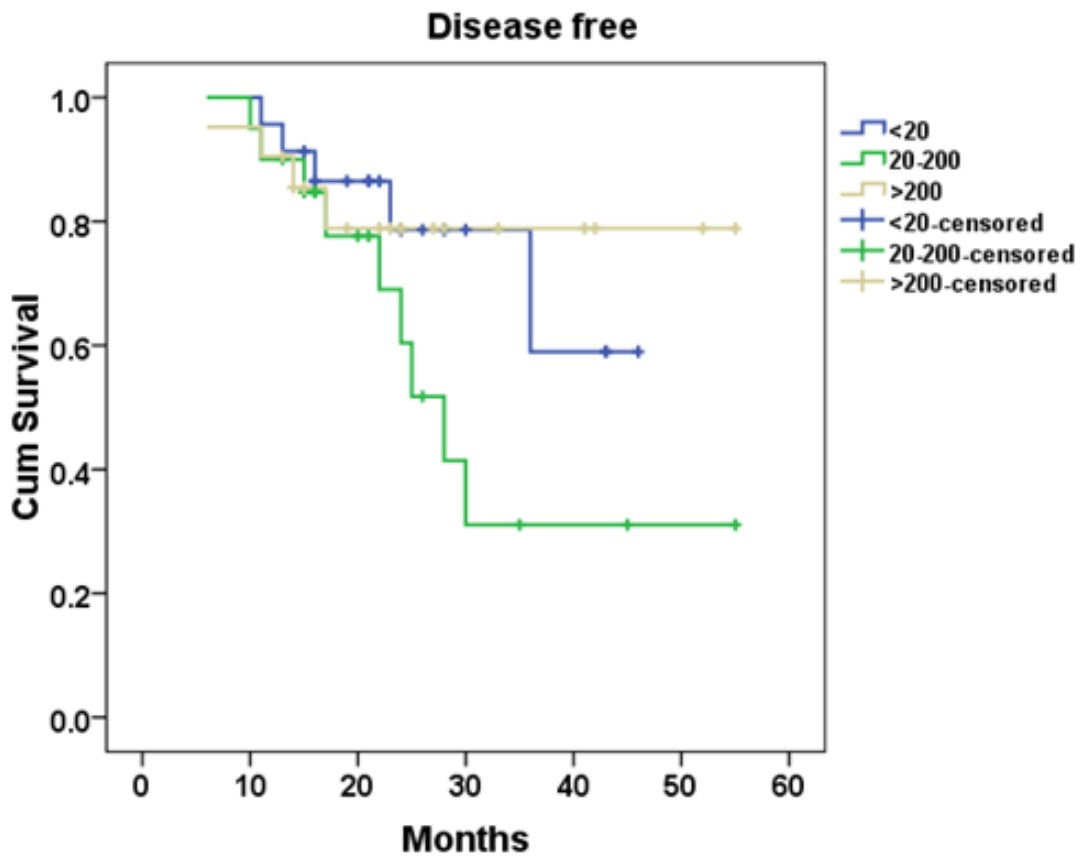

Figure (2): Kaplan-Meier survival curves showing the disease-free survival of the 153 studied patients subdivided according to their alpha-fetoprotein serum levels at the diagnosis of HCC $(<20 \mathrm{ng} / \mathrm{ml} ; 20$ $200 \mathrm{ng} / \mathrm{ml} ;>200 \mathrm{ng} / \mathrm{ml}$ ) 


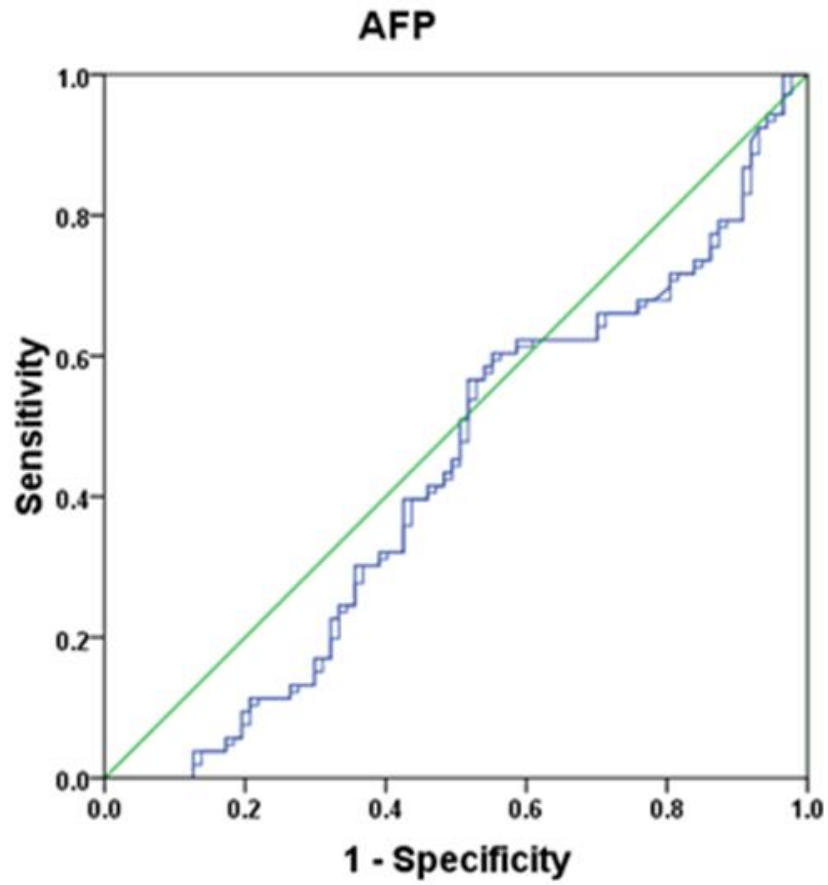

Figure (3): ROC curve showing the overall accuracy of alpha-fetoprotein serum levels for discriminating between survivors and deceased patients.

\section{Recurrence}

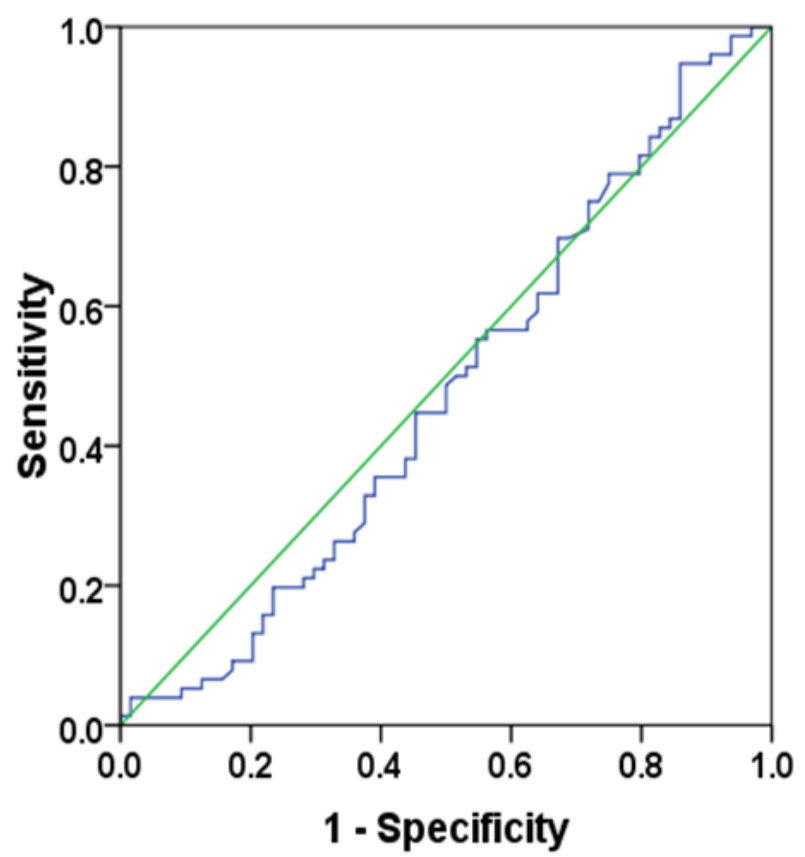

Figure (4): ROC curve showing the overall accuracy of alpha-fetoprotein serum levels for discriminating between recurrence and non-recurrence patients. 


\section{DISCUSSION}

Serum AFP levels tend to be nonspecifically elevated in patients with chronic liver disease, and the diagnostic levels of this tumor marker are seldom observed in patients with small HCCs [1].

Consequently, updated AASLD guidelines for the diagnosis and management of HCC dropped AFP as a routine marker for HCC surveillance in patients with cirrhosis, but this decision was debatable [3].

Several studies have reported the ability of AFP response to predict response to therapy and survival outcomes [7]. However, there is no consensus yet regarding the magnitude of the decrease in AFP that defines AFP response [8].

In a systematic review of 72 studies of prognostic markers in HCC, Tandon and Garcia-Tsao [4] identified AFP as one of the most robust prognostic indexes, although they noticed that the appropriate cutoff level and the group of patients in which this serum marker may be beneficial has to be established. Thus, we deemed it of interest to evaluate the prognostic value of AFP in patients who might benefit most from the curative treatment and, therefore, are those for whom prognostication should be of utmost importance.

Among cases treated with RFA, HCC recurred in 88 cases $(57.5 \%)$ in the current study. Recurrence of HCC after RFA is neither uncommon nor specific to this therapy. Even patients treated with hepatic resection showed recurrence rates $>70 \% 5$ years after surgery [9].

A large study demonstrated that the etiology of liver disease is an important predictor of longterm survival and distant intrahepatic recurrence after RFA and identified a role for chronic hepatitis $\mathrm{C}$ virus (HCV) in survival [10]. In another study, it was shown that patients with HCV-related cirrhosis who achieved sustained virological response to antiviral therapy have a substantially lower rate of HCC recurrence and accompanying higher survival rate [11].

$92.8 \%$ of the patient cohort in our study was HCV-positive and this status may have played a role in the high recurrence rate.

In the present study, regarding overall survival, Child class A patients could achieve a better survival after RFA than those with Child class B $(\chi 2=34.613, P=0.000)$. Similar to our results, Lee et al. [12] found that Child-Pugh class B (relative risk $=2.43, \mathrm{P}=.011$ ) is one of the significant predictive factors for poor overall survival.

The development and recurrence of HCC may be attributed to the severity of the underlying liver disease, and thus reinforces the importance and the role of liver function in hepato-carcinogenesis [11].

Kikuchi et al. [13] agree with our results as they found that the survival was associated with the Child-Pugh only at a statistically significant level using multiple Cox regression analysis.

Because most HCCs arise in the context of liver cirrhosis, the established liver dysfunction may already represent generally a poor prognosis. Indeed, HCC treatment has no impact on the outcome for Child-Pugh class C patients [3].

In patients with Child-Pugh class B, however, HCC treatment can be beneficial, but the outcome is not consistent. Asymptomatic HCC patients and without decompensated cirrhosis are categorized as patients with ascites, encephalopathy, and/or coagulopathy [13]. In the present study, ChildPugh class B patients comprised $32.1 \%$ of the study population.

Recently in 2017, although performed on patients after hepatectomy, Shinozuka et al. [14] concluded that Child-Pugh class (A or B) before RFA was a significant predictor of long-term survival.

In the present study, Kaplan-Meier overall survival curves of the studied patients, who were subdivided according to their lesion size at the time of diagnosis of $\mathrm{HCC}(<3$ and $>/=3 \mathrm{~cm})$, revealed no statistically significant difference $(\chi 2=0.305, \mathrm{P}=0.581)$. This finding is consistent with the results of Giannini et al. [15] who found that there was no significant survival difference associated with the size of the HCC $(\leq 2$ or $2-3$ $\mathrm{cm})$.

In the present study, the survival rate at 5 years was $48.8 \%$, while in the study by Giannini et al. [15] the 5-year survival rate was approximately $60 \%$ in both patients with AFP serum levels below and above $200 \mathrm{ng} / \mathrm{ml}$. The higher survival rate in the study of Giannini et al. [15] comes from the different study population. In their study, patients with compensated liver cirrhosis (ChildPugh class A) were included and an Eastern Cooperative Oncology Group Performance Status of 0 who were diagnosed with a single, small (i.e., $\leq 3 \mathrm{~cm}) \mathrm{HCC}$, and they used all curative modalities including orthotopic liver transplantation, hepatic resection, percutaneous ethanol injection and 
RFA. In our study, we included patients with Child class A and B, and approximately $80 \%$ of our patients had lesions $\geq 3 \mathrm{~cm}$ and all our patients were treated with RFA only.

It seems that the predictive ability of AFP depends mainly on tumor size and treatment modality, being more evident in patients with advanced HCC and in those who received a palliative treatment, and less evident in patients with small tumors and in those who underwent curative treatment [16].

Indeed, the prognostic role of AFP was dramatically diluted in studies excluding patients with advanced liver disease and/or advanced HCC [17]. These considerations are also supported by the evidence in our series that there was no "therapeutic disparity," and that causes of death were evenly distributed across patients with normal, mildly, and markedly elevated AFP levels, likely ruling out the presence of other possible prognostic confounding factors.

In some studies, it was shown that the rate of increase in serum AFP levels may have prognostic role in HCC patients awaiting liver transplantation; yet, these studies couldn't identify a role for static AFP levels as a predictor of survival or HCC recurrence after liver transplantation $[\mathbf{1 8 , 1 9 ]}$.

Overall survival (time to death or end of the study in months), was $95 \%, 75.6 \%, 55.6 \%, 48.8 \%$, and $48.8 \%$ at $1,2,3,4$ and 5 years respectively. The mean survival interval was 33.6 months in group $1,34.3$ months in group 2, and 28.6 months in group 3 with no significant differences among the three groups $(\mathrm{p}=0.207)$.

In the study by Farinati et al. [20], the mean survival time for the group of treated patients with AFP levels ( $<20 \mathrm{ng} / \mathrm{ml})$ was 39 months, while it was 31 months and 20 months for the patients with AFP levels of (21- $400 \mathrm{ng} / \mathrm{ml})$ and $(>400$ $\mathrm{ng} / \mathrm{ml}$ ), respectively, with a significant link between AFP level and survival time.

This difference could be attributed to several factors, such as the larger study population in the study of Farinati et al. [20]. Additionally, their study population was not homogenously distributed among the three groups as patients were subdivided into 3 AFP groups: normal $(<20$ $\mathrm{ng} / \mathrm{ml}$ ) [46\% of the study population], elevated $(21-400 \mathrm{ng} / \mathrm{ml})$ [36\% of the study population], and diagnostic $(>400 \mathrm{ng} / \mathrm{ml})[18 \%$ of the study population].

In the present study, the ROC curve showed that AFP had inadequate accuracy in discriminating survivors and deceased patients (AUC 0.435, 95\% CI= 0.338-0.531). These results are consistent with that of Giannini et al. [15] (AUC 0.536, $95 \% \mathrm{CI}=0.465-0.606$ ).

From our results, we demonstrated that AFP level could not be used as a good predictor of either death or recurrence. This finding agrees with the study by Kiriyama et al. [21] who found that serum AFP levels did not have value in predicting recurrence or death. Shim et al. [22] found that the time-dependent risks of recurrence and cancerspecific death were similar in patients with AFPproducing $\mathrm{HCC}$ and AFP-nonproducing $\mathrm{HCC}$ who were treated by liver resection.

In contrast to our results, it was reported by Park et al. [23] that patients who showed an AFP response had significantly longer overall survival and progression-free survival than AFP non-responders.

Contrary to our results, a recent study by Zhang et al. [24] concluded that tumor size, albumin, prothrombin time, and $\alpha$ - fetoprotein levels were independently associated with mortality after RFA for HCC, while tumor size and HBV-DNA were independently associated with recurrence.

The difference between our results and those of Zhang et al. [24] can be attributed to many factors. First, they included only patients with high AFP before treatment, while we included all patients with different levels. Second, the viral status of their patients was HBV, while in our study; approximately $93 \%$ of our patients were HCVpositive.

The prognostic role of alpha-fetoprotein reported in other studies may be due to the heterogeneous liver and tumor-related characteristics, as well as different modalities of HCC treatment in the studied populations [9].

A major limitation of the current study is that it is a retrospective study with a relatively small number of patients. Furthermore, the feasibility of RFA is mainly dependent on the operator's technique, the experience, and the equipment available at the center. Moreover, the findings in the current study were obtained from a singlecenter cohort and cannot be compared to clinical experience at other treatment centers, due to the heterogeneity of selection and patient management, physician expertise, the indication for additional treatments, and the institution's volume of care.

In conclusion, our results demonstrated that AFP level could not be used as a good predictor of either death or recurrence after RFA in HCC cases. 
Conflicting Interest: No conflict of interest.

Institutional Review Board Statement and Ethical Committee Approval: This study involved human participants and was reviewed and approved by the Ethics Committee of Ain Shams University Hospitals.

Informed consent statement: Patients were not required to give informed consent to the study because the records of patients were reviewed in a retrograde manner and the clinical data that were obtained after each patient agreed to treatment by written consent.

\section{REFERENCES}

1. Sterling RK, Wright EC, Morgan TR, Seeff LB, Hoefs JC, Di Bisceglie AM, et al. Frequency of elevated hepatocellular carcinoma (HCC) biomarkers in patients with advanced hepatitis $\mathrm{C}$. Am J Gastroenterol 2012; 107: 64-74.

2. Yamashita T, Forgues M, Wang W, Kim JW, Ye $\mathrm{Q}$, Jia $\mathrm{H}$, et al. EpCAM and $\alpha$-fetoprotein expression defines novel prognostic subtypes of hepatocellular carcinoma. Cancer Res 2008; 68: 1451-61.

3. Bruix J, Sherman M;,American Association for the Study of Liver Diseases. Management of hepatocellular carcinoma: an update. Hepatology 2011; 53: 1020-2.

4. Tandon P, Garcia-Tsao G. Prognostic indicators in hepatocellular carcinoma: a systematic review of 72 studies. Liver Int 2009; 29: 502-10.

5. Huo TI, Lee SD. Role of the model for end-stage liver disease and serum a-fetoprotein as predictors for hepatocellular carcinoma. Liver Int 2006; 26: 1300-1.

6. Lencioni R, Llovet JM. Modified RECIST (mRECIST) assessment for hepatocellular carcinoma. Semin Liver Dis 2010; 30: 52-60

7. Memon K, Kulik L, Lewandowski RJ, Wang E, Ryu RK, Riaz A, et al. Alpha-fetoprotein response correlates with EASL response and survival in solitary hepatocellular carcinoma treated with transarterial therapies: A subgroup analysis. $J$ Hepatol 2012; 56: 1112-1120

8. Yau T, Yao TJ, Chan P, Wong H, Pang R, Fan ST, et al. The significance of early alphafetoprotein level changes in predicting clinical and survival benefits in advanced hepatocellular carcinoma patients receiving sorafenib. Oncologist 2011; 16: 1270-1279

9. Kaibori M, Kubo S, Nagano H, Hayashi M, Haji $\mathrm{S}$, Nakai T, et al. Clinicopathological features of recurrence in patients after 10-year disease-free survival following curative hepatic resection of hepatocellular carcinoma. World J Surg 2013; 37: 820-828.

10. Shiina S, Tateishi R, Arano T, Uchino K, Enooku $\mathrm{K}$, Nakagawa $\mathrm{H}$, et al. Radiofrequency ablation for hepatocellular carcinoma: 10-year outcome and prognostic factors. Am J Gastroenterol 2012; 107:569-577.

11. N'Kontchou G, Mahamoudi A, Aout M, GanneCarrié N, Grando V, Coderc E, et al. Radiofrequency ablation of hepatocellular carcinoma: long-term results and prognostic factors in 235 Western patients with cirrhosis. Hepatology 2009; 50: 1475-1483.

12. Lee DH, Lee JM, Lee JY, Kim SH, Yoon JH, Kim YJ, et al. Radiofrequency Ablation of Hepatocellular Carcinoma as First-Line Treatment: Long-term Results and Prognostic Factors in 162 Patients with Cirrhosis. Radiology 2014; 270: 900-9.

13. Kikuchi L, Menezes M, Chagas AL, Tani CM, Alencar RS, Diniz MA, et al. Percutaneous radiofrequency ablation for early hepatocellular carcinoma: Risk factors for survival. World $J$ Gastroenterol 2014; 20: 1585-1593.

14. Shinozuka K, Shibata T, Imamine R, Kataoka M and Togashi K. Effectiveness of Radiofrequency Ablation of Initial Recurrent Hepatocellular Carcinoma after Hepatectomy: Long-Term Results and Prognostic Factors. Open Journal of Radiology 2017; 7: 177-189.

15. Giannini EG, Marenco S, Borgonovo G, Savarino V, Farinati F, Del Poggio P, et al. AlphaFetoprotein Has No Prognostic Role in Small Hepatocellular Carcinoma Identified During Surveillance in Compensated Cirrhosis. Hepatology 2012; 56: 1371-9.

16. Pompili M, Rapaccini GL, Covino M, Pignataro G, Caturelli E, Siena DA, et al. Prognostic factors for survival in patients with compensated cirrhosis and small hepatocellular carcinoma after percutaneous ethanol injection therapy. Cancer 2001; 92: 126-135.

17. Nomura F, Ohnishi K, Tanabe Y. Clinical features and prognosis of hepatocellular carcinoma with reference to serum alpha-fetoprotein levels. Analysis of 606 patients. Cancer 1989; 64: 17001707.

18. Vibert E, Azoulay D, Hoti E, Iacopinelli S, Samuel D, Salloum C, et al. Progression of alphafetoprotein before liver transplantation for hepatocellular carcinoma in cirrhotic patients: a critical factor. Am J Transplant 2010; 10: 129137.

19. Merani S, Majno P, Kneteman NM, Berney T, Morel P, Mentha G, et al. The impact of waiting 
list alpha-fetoprotein changes on the outcome of liver transplant for hepatocellular carcinoma. $J$ Hepatol 2011; 55: 814-819.

20. Farinati F, Marino D, De Giorgio M, Baldan A, Cantarini M, Cursaro C, et al. Diagnostic and Prognostic Role of alpha-Fetoprotein in Hepatocellular Carcinoma: Both or Neither? Am J Gastroenterol 2006; 101: 524-532.

21. Kiriyama S, Uchiyama $\mathrm{K}$, Ueno $\mathrm{M}$, Ozawa $\mathrm{S}$, Hayami S, Tani M, et al. Triple positive tumor markers for hepatocellular carcinoma are useful predictors of poor survival. Ann Surg 2011; 254: 984-991.

22. Shim JH, Yoon DL, Han S, Lee YJ, Lee SG, Kim $\mathrm{KM}$, et al. Is Serum Alpha-Fetoprotein Useful for
Predicting Recurrence and Mortality Specific to Hepatocellular Carcinoma After Hepatectomy? A Test Based on Propensity Scores and Competing Risks Analysis. Ann Surg Oncol 2012; 19: 36873696.

23. Park H, Kim SU, Park JY, Kim DY, Ahn SH, Chon CY, et al. Clinical usefulness of double biomarkers AFP and PIVKA-II for subdividing prognostic groups in locally advanced hepatocellular carcinoma. Liver International 2014; 34 : 313-321.

24. Zhang W, Luo E, Gan J, Song X, Bao Z, Zhang $\mathrm{H}$, et al. Long-term survival of hepatocellular carcinoma after percutaneous radiofrequency ablation guided by ultrasound. World J Surg Oncol 2017; 15: 122-128. 